\title{
Article
}

\section{Electrospinning predictions using artificial neural networks}

Brooks, Hadley Laurence and Tucker, Nick

Available at http://clok.uclan.ac.uk/12183/

Brooks, Hadley Laurence ORCID: 0000-0001-9289-5291 and Tucker, Nick (2015) Electrospinning predictions using artificial neural networks. Polymer, 58 . pp. 22-29. ISSN 0032-3861

It is advisable to refer to the publisher's version if you intend to cite from the work. http://dx.doi.org/10.1016/j.polymer.2014.12.046

For more information about UCLan's research in this area go to http://www.uclan.ac.uk/researchgroups/ and search for < name of research Group>.

For information about Research generally at UCLan please go to http://www.uclan.ac.uk/research/

All outputs in CLoK are protected by Intellectual Property Rights law, including Copyright law. Copyright, IPR and Moral Rights for the works on this site are retained by the individual authors and/or other copyright owners. Terms and conditions for use of this material are defined in the policies page.

\section{CLoK}

Central Lancashire online Knowledge www.clok.uclan.ac.uk 
Title: Electrospinning predictions using artificial neural networks

Authors: Hadley Brooksa, Nick Tucker

aSchool of Engineering, University of Central Lancashire, hlbrooks@uclan.ac.uk

Abstract:

Electrospinning is a relatively simple method of producing nanofibres. Currently there is no method to predict the characteristics of electrospun fibres for a wide range of polymer/solvent combinations and concentrations without first measuring a number of solution properties. This paper shows how artificial neural networks can be trained to make electrospinning predictions using only commonly available prior knowledge of the polymer and solvent. Firstly, a probabilistic neural network was trained to predict the classification of three possibilities; no fibres (electrospraying), beaded fibres and smooth fibres with over $80 \%$ correct predictions. Secondly, a generalised neural network was trained to predict fibre diameter with an average absolute percentage error of $22.3 \%$ for the validation data. These predictive tools can be used to reduce the parameter space prior to scoping exercises.

Graphical Abstract:

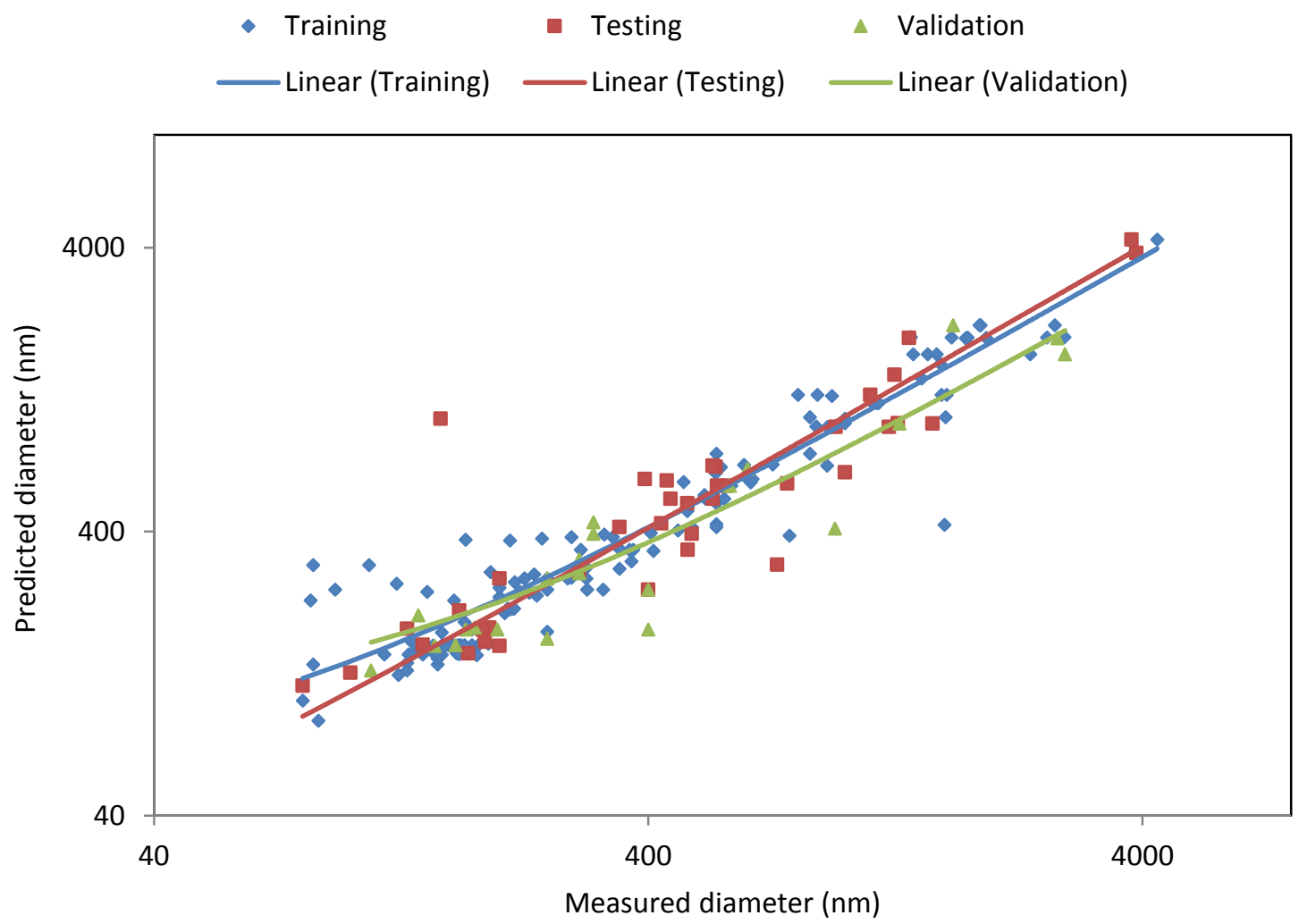

Predicted versus measured electrospun-fibre diameter from an artificial neural network trained using data from 13 different polymer/solvent combinations from over 20 independent studies. 
Keywords: Artificial Neural Network, Electrospinning, Prediction, Classification, Diameter, Nanofibre.

1. Introduction:

Electrospinning is an increasingly important area of research as developments in biotechnology, electronics, catalysts, composites and sensors all call for increased flexibility and control over the manufacture of nano-scale fibres [1, 2]. Specific applications include tissue scaffolds [3-11], membranes for fuel cells [12], separation layers for batteries [13], air and water filtration [14, 15], reinforcement for nano-composites [2, 16-18], piezoelectric fibres for energy harvesting and sensors [19-21] and conductive layers in solar cells and electronics [17, 21, 22]. Electrospinning compares favourably with other methods of nano-fibre manufacture such as drawing, template synthesis, phase separation and self-assembly due to its relative simplicity and low cost [23-26].

Needle or capillary electrospinning of polymer solutions typically works by drawing a small electrostatically charged jet from a droplet to a grounded collector (Figure 1). The jet is initiated from the droplet when the repelling forces of the surface charges overcome the surface tension and viscous forces of the droplet. The jet diameter reduces as it is stretched and the solvent evaporates. At a certain point, known as the whipping instability, the electrostatic repulsion overcomes the viscoelastic forces and the jet flight path changes to an expanding helix causing further stretching and drying of the fibre. In most cases this leads to dry non-woven nano-fibres depositing on the collector [27].

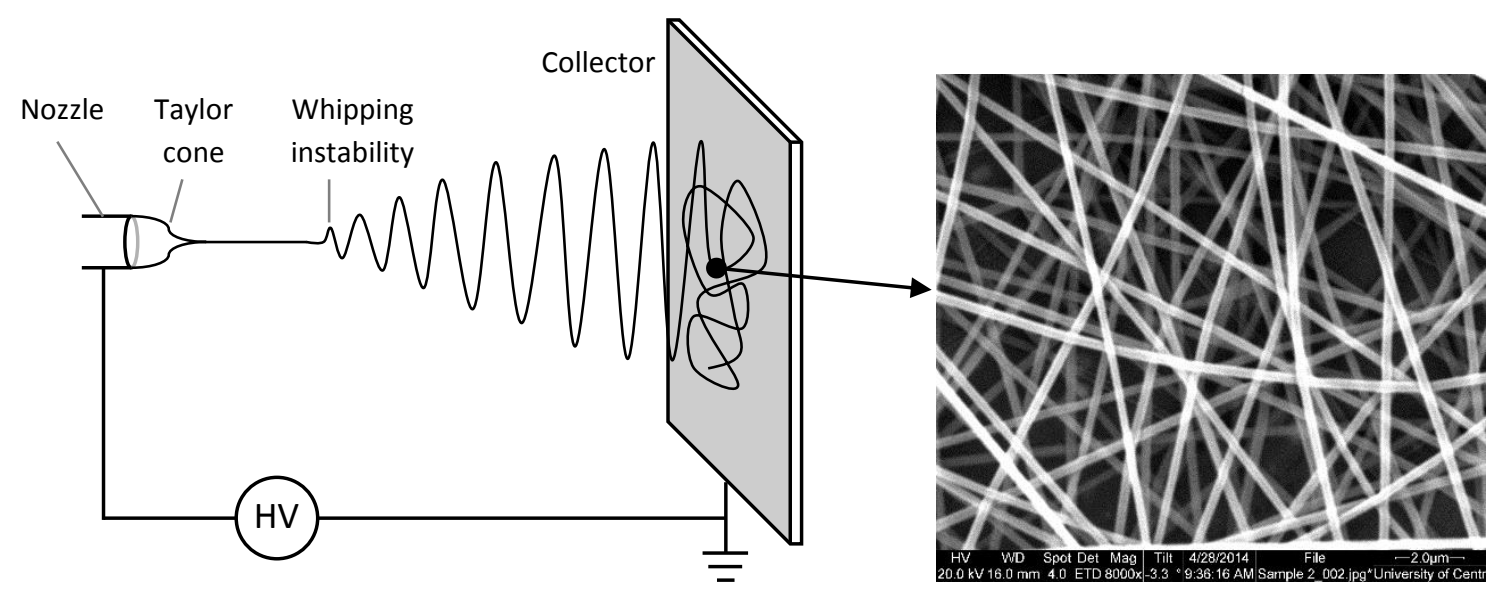

Figure 1. Schematic of a typical electrospinning setup and an SEM of electrospun nanofibres.

Many groups have studied the effects of electrospinning parameters on fibre characteristics with the aim of optimising fibres for specific applications. Koski et al. studied the effect of molecular weight and polymer concentration for poly(vinyl alcohol) (PVOH) and water systems. Electrospinning was found to occur if the product of the intrinsic viscosity and polymer concentration was over a certain threshold [28]. This supports the theory that the level of polymer chain entanglement dictates whether electrospinning or electrospraying dominates. Son et al. studied the effect of various solvents and polyelectrolytes on poly(ethylene oxide) (PEO) fibre diameter and morphology. A nonlinear inverse relationship was found between the dielectric constant of the solvent and the fibre diameter [29]. Theron et al., Cui et al., Thomson et al. and Coles et al. all carried out parameter 
studies for various polymer/solvent systems [30-33]. While it is difficult to directly compare findings, certain trends seem to be universal i.e. the qualitative relationships between polymer concentration and resulting fibre diameter.

Building on from similar parameter studies empirical models have been created with good predictive capabilities, albeit for specific polymer/solvent and equipment combinations. Yördem et al. and Gu et al. used response surface methodology (RSM) to predict poly(acrylonitrile) (PAN) fibre diameters based on chosen parameters. They found polymer concentration to have a significantly larger effect than voltage and distance [34,35]. These studies aim to optimise polymer/solvent systems which were initially found using trial and error. These trial and error scoping exercises are time intensive, costly and ideally could be replaced with generalised predictive empirical or analytical models. Currently there is no way to predict the characteristics of new electrospun materials based on prior knowledge of the polymer and solvent alone.

Accurate analytical modelling of fibre formation is complicated due to the nonlinear relationships in electrohydrodynamics with solvent loss. In addition to this many material properties such as the intrinsic viscosity and bulk/surface charge density need to be determined experimentally before being incorporated into the models [32, 36-41]. Notwithstanding these difficulties, important models have been validated that describe different portions of the jet for selected materials. Taylor introduced the "Leaky Dielectric Model" in the 1960's which describes how the surface charges of the droplet are balanced with the tangential viscous forces of the liquid resulting is what is known as the Taylor cone [42]. In the 1970's Saville studied the stability of fluid cylinders in a longitudinal electric field [43]. Reneker et al. created a mathematical model to explain the whipping instability based on the rheological properties of the polymer solution and lateral perturbations $[32,36,41$, 44]. Hohman et al. developed mathematical models for three different instabilities and used the models to create operating diagrams for when electrospinning occurs [37-39]. Feng created a 1D model based on slender body theory to examine the role of nonlinear rheology on the electrically stretched jet [40].

Despite the aforementioned empirical and analytical models there has been little work into predictive methods that are generalised and do not require a priori measurements of the solution properties. Helgeson and Wagner developed a generalised predictive model for fibre diameters using dimensional analysis and measured parameters such as solution conductivity and dry fibre diameter. The predictions worked well for electrospinning parameters resulting in smooth fibres but deviated slightly for parameters where beading is known to occur [45].

Sarkar et al. and Klanlou et al. trained artificial neural networks (ANNs) to predict fibre diameters for PEO and poly(methyl methacrylate) (PMMA) respectively [46, 47]. Sarkar was able to predict fibre diameters over several datasets taken from literature; however this data contained a priori solution measurements. Nasouri et al. used a response surface methodology (RSM) and artificial neural networks (ANNs) to predict the highest production rate for PAN nanofibres within $4 \%$ of the measured value [48]. In all cases where RSM and ANNs were compared the authors noted ANNs were better able to predict electrospinning characteristics $[47,48]$.

In this study ANNs are used to create generalised empirical models for the prediction of electrospun fibre diameters and morphology based on literature data. Specifically this includes function approximation for fibre diameter prediction and classification to predict which category (fibre, 
beaded fibre or no fibre) will prevail given a specific polymer/solvent combination. The primary aim is to determine whether an ANN trained only with data containing a priori information can compete with ANNs trained with data containing solution measurements for diameter and morphology prediction.

\section{Process parameters:}

Studies of the electrospinning process have shown that to understand the phenomenon a large number of material and experimental parameters are required. Table 1 shows a list of parameters that affect the electrospinning process, including a qualitative measure of their influence on the fibre diameter and whether that parameter is known a priori, is an independent variable, or needs to be measured. Where conflict exists between findings in the literature preference was given to experimental findings over theoretical models.

Table 1. Electrospinning parameters including their influence of fibre diameter and morphology.

\begin{tabular}{|c|c|c|c|}
\hline $\begin{array}{l}\text { Parameter } \\
\text { group }\end{array}$ & Parameter & $\begin{array}{c}\text { Impact inferred from } \\
\text { literature }\end{array}$ & $\begin{array}{l}\text { Easily known } \\
\text { or controlled }\end{array}$ \\
\hline \multirow{7}{*}{ Polymer } & Molecular weight & Strong $[28,30,31,49]$ & $\checkmark$ \\
\hline & Polydispersity & Moderate [50-52] & $x$ \\
\hline & Intrinsic viscosity & Strong $[45,51]$ & $x$ \\
\hline & Density & Weak [32] & $\checkmark$ \\
\hline & Glass transition temperature & Unknown & $\checkmark$ \\
\hline & Dielectric constant & Unknown & - \\
\hline & Solubility parameters & Strong [53] & $x$ \\
\hline \multirow{7}{*}{ Solvent } & Density & Weak [32] & $\checkmark$ \\
\hline & Zero shear viscosity & Moderate [45] & $\checkmark$ \\
\hline & Vapour pressure & Moderate $[32,54]$ & $\checkmark$ \\
\hline & Dielectric constant & Strong $[29,53]$ & $\checkmark$ \\
\hline & Conductivity & Strong $[30,33,46,55]$ & $\checkmark$ \\
\hline & Dipole moment & Strong [54] & $\checkmark$ \\
\hline & Surface tension & Weak $[30,32,44]$ & $\checkmark$ \\
\hline \multirow{7}{*}{ Solution } & Concentration & Strong $[28,30,33,34,49,51]$ & $\checkmark$ \\
\hline & Relaxation time & Strong [32] & $x$ \\
\hline & Viscosity & Strong $[30,32,40,41]$ & $x$ \\
\hline & Vapour pressure & Moderate [32] & $x$ \\
\hline & Conductivity & Strong $[30,33,46,55]$ & $x$ \\
\hline & Surface tension & Weak $[30,32,44]$ & $x$ \\
\hline & Charge density & Strong $[30,32,38]$ & $x$ \\
\hline \multirow{7}{*}{ Experimental } & Feed rate & Weak $[39,45,49,56]$ & $\checkmark$ \\
\hline & Nozzle geometry & Weak [56-58] & $\checkmark$ \\
\hline & Distance & Weak $[30,33,49,56]$ & $\checkmark$ \\
\hline & Voltage & Moderate $[34,35,56,59]$ & $\checkmark$ \\
\hline & Atmospheric humidity & Weak $[32,50,60]$ & $x$ \\
\hline & Atmospheric temperature & Weak [60] & $x$ \\
\hline & Collector geometry & Moderate $[56,59]$ & $\checkmark$ \\
\hline
\end{tabular}


From the number of parameters shown in table 1, which is not exhaustive, it is clear why analytical modelling of the electrospinning process is difficult. In contrast with this is the relative ease in which electrospun fibres can be produced in the laboratory for a wide range of polymer/solvent systems. Insight into this contradiction can be gained by focussing only on the few parameters which have the strongest influence and by knowledge of the interconnected nature of the parameters.

The following sections will provide rationale for which parameters will be included in the training data. An attempt will be made to suggest which 'hard to measure' parameters may be replaced with parameters that are known a priori or are independently variable.

The method proposed in this paper is to find empirical models using statistical techniques. Quantitative relationships between the solution properties and the polymer or solvent properties will not be known in advance as it is hypothesised that the strongest interdependencies will be encapsulated in the trained ANNs.

\subsection{Polymer properties}

Arguably, the most important polymer parameter affecting electrospinning is the molecular weight. The length and size of the molecules directly influence the intrinsic viscosity of the polymer in solution as described by the Mark-Houwink equation [28, 51]. The higher the molar mass the lower the critical concentration needs to be before electrospinning can occur. The molecular weight has also been shown to have power law relationships with fibre diameter. Due to the fact the molecular weight is largely known for a given polymer and intrinsic viscosity is not, it is suggested that molecular weight may be used to supplant viscosity measurements in an empirical predictive model.

The influence of polydispersity (or molecular weight distribution) on viscosity (and hence fibre diameter) is moderate [50-52]. As this information is often not known it will not be directly included in this work and will provide a known source of error.

In an attempt to provide the ANN with information about the polymer chain structure the inclusion of glass transition temperature data will be assessed. The glass transition temperature for a given polymer with a constant molecular weight is known to vary with the degree of branching [61]. The glass transition temperature combined with the molecular weight and the polymer concentration may help to improve the predicted level of polymer chain entanglement and thus solution viscosity.

To the authors' knowledge the influence of the polymers dielectric constant in relation to electrospinning has not been studied. The dielectric constant is a measure of the polymers ability to polarise in the presence of an electric field. It is therefore also an indirect measure of the polymer chains polarity. The polarity of a polymer strongly influences whether it is soluble in a solvent, following the well-known rule of thumb 'like dissolves like'. The effect of solubility on the critical entanglement and fibre diameter is related to the hydrodynamic volume of the solute, which is larger in good solvents [51]. Therefore the solubility of any polymer/solvent system should be incorporated (directly or indirectly) into any predictive model. Polymer and solvent solubility parameters, such as Hildebrand or Hansen solubility parameters could also be useful, but will not be used in this study.

\subsection{Solvent properties}


The zero shear viscosity is known for most common solvents and together with the intrinsic viscosity of the polymer and the polymer concentration contributes to the solution viscosity.

Solvent evaporation has an important influence on fibre morphology and is related to parameters such as vapour pressure and boiling point.

The conductivity of a solution depends on complicated interactions between the solute and the solvent. The presence of small quantities of impurities or electrolytes can have a significant effect on conductivity. However, as this parameter needs to be measured the solvents conductivity will be used to provide a predictor of solution conductivity.

The surface tension of the solvent is considered a critical parameter in many theoretical models, however experimentally it has been found to have a weak influence on fibre diameter [30,32], while it may impact highly on fibre morphology [44]. For this reason the ANN will be trained with solvent surface tension values verify their impact.

\subsection{Solution properties}

The most important solution property is concentration, as together with molecular weight it determines whether or not there is a critical level of molecular entanglement for electrospinning to occur $[28,30,33,35,51]$. The concentration also follows power law relationships with fibre diameter and can easily be varied prior to electrospinning.

It is hypothesised that many of the other solution properties such as surface tension, vapour pressure, dielectric constant, charge density, conductivity and viscosity can be derived (at least in principle) from the polymer and solvent parameters.

\subsection{Experimental parameters}

The electrospinning voltage has a moderate effect on fibre diameter as it is responsible for the drawing of the jet from the Taylor cone. In literature this is often reported as nominal electric field strength in $\mathrm{kV} / \mathrm{cm}$, the distance referring to the gap between the nozzle tip and collector [39, 40, 46].

There are conflicting reports on the significance of flow rate on the fibre diameter in the literature. Some report the flow rate is a parameter that can be used to control the final fibre diameter whilst others report it has no statistical significance [30, 39, 46, 49, 62]. In the case of stable electrospinning where the meniscus at the spinning tip maintains constant shape, the flow rate into the Taylor cone must balance the rate of drawing from the jet. Over-feeding causes the meniscus to swell and fall off the nozzle tip while lower flow rates result in starvation and stuttering of the jet. Unstable flow is likely to produce a wider variation in fibre diameters, however, there is little evidence to suggest a significant effect on the average fibre diameter $[49,63]$. In this study the effect of flow rate will be assumed to be weak and thus not essential for the predictive model. Experiments have shown the nozzle diameter to have a statistically weak effect on fibre diameter and so will not be included in this study [56-58].

Typically single needle electrospinning is carried out at room temperature at atmospheric pressure and humidity. Whilst atmospheric conditions are known to contribute to fibre diameter and morphology, unless these conditions vary significantly from standard atmospheric conditions, the 
effects are minimal $[32,50,60]$. Additionally these parameters are often not quoted in the literature and are difficult to control with most laboratory setups. For these reasons standard laboratory atmospheric conditions will be assumed in this study.

\subsection{Selected parameters}

Table 2 summarises which parameters will be focussed on for the development of the predictive models. All effort has been made to focus on parameters known to have strong or moderate impacts on electrospinning characteristics, are known in advance or can be independently varied. Some parameters thought to have weak impacts will be included for confirmation.

Table 2. Parameters selected to be trialled in the predictive models.

\begin{tabular}{|c|c|c|c|}
\hline $\begin{array}{l}\text { Parameter } \\
\text { group }\end{array}$ & Parameter & $\begin{array}{c}\text { Impact inferred from } \\
\text { literature }\end{array}$ & $\begin{array}{c}\text { Easily } \\
\text { known or } \\
\text { controlled }\end{array}$ \\
\hline \multirow{3}{*}{ Polymer } & Molecular weight, $\left(\mathrm{M}_{\mathrm{w}}\right)$ & Strong $[28,30,31,49]$ & $\checkmark$ \\
\hline & Glass transition temperature, $\left({ }^{\circ} \mathrm{C}\right)$ & Unknown & $\checkmark$ \\
\hline & Dielectric constant at $1 \mathrm{MHz}$ & Unknown & - \\
\hline \multirow{8}{*}{ Solvent } & Density, $(\mathrm{g} / \mathrm{ml})$ & Weak [32] & $\checkmark$ \\
\hline & Zero shear viscosity, (cps) & Moderate [45] & $\checkmark$ \\
\hline & Vapour pressure, (Torr) & Moderate $[32,54]$ & $\checkmark$ \\
\hline & Dielectric constant & Strong $[29,53]$ & $\checkmark$ \\
\hline & Dipole moment, (D) & Strong [54] & $\checkmark$ \\
\hline & Boiling point, $\left({ }^{\circ} \mathrm{C}\right)$ & Unknown & $\checkmark$ \\
\hline & Conductivity, $(\mathrm{S} / \mathrm{cm})$ & Strong $[30,33,46,55]$ & $\checkmark$ \\
\hline & Surface tension, $(\mathrm{mN} / \mathrm{m})$ & Weak $[30,32,44]$ & $\checkmark$ \\
\hline \multirow{2}{*}{ Solution } & Concentration, $\left(\%_{w / v}\right)$ & Strong $[28,30,33,34,49,51]$ & $\checkmark$ \\
\hline & Electric field strength, $(\mathrm{kV} / \mathrm{cm})$ & Moderate $[39,40,43,46,64]$ & $\checkmark$ \\
\hline
\end{tabular}

3. Experimental data:

The data used to create the predictive models was gathered from literature as shown in Tables 3 and 4. Only data relating to single needle/capillary electrospinning of primary solvent systems was used. Differences in needle geometry, flow rate, collector geometry and atmospheric conditions are not taken into account and are a known source of error. Effort was made to avoid including tests which involved the effects of electrolytes to change solution conductivity. Common solvent properties were taken from literature [65].

Table 3. Data from literature used to train and test the ANN diameter prediction models.

\begin{tabular}{|c|l|c|c|l|}
\hline Polymer & \multicolumn{1}{|c|}{ Solvent } & Fibre diameter $(\mathrm{nm})$ & Data points & Reference \\
\hline PS & DMF & $292-4284$ & 21 & {$[55]$} \\
\hline PVOH & Water & $192-731$ & 31 & {$[33,66]$} \\
\hline PEO & Water & $80-540$ & 18 & {$[46]$} \\
\hline \multirow{2}{*}{$P L A$} & DCM & $200-1600$ & 16 & {$[67]$} \\
\cline { 2 - 5 } & Acetone & $86-1560$ & 6 & {$[31]$} \\
\hline \multirow{3}{*}{$P V B$} & Ethanol & $130-1000$ & 6 & {$[68]$} \\
\cline { 2 - 5 } & Isopropanol & $130-350$ & 5 & {$[68]$} \\
\cline { 2 - 5 } & n-Butanol & $110-130$ & 3 & {$[68]$} \\
\hline
\end{tabular}




\begin{tabular}{|c|l|c|c|c|}
\hline PA 66 & Formic acid & $117-198$ & 38 & {$[69]$} \\
\hline PAN & DMF & $83-2782$ & 51 & {$[34]$} \\
\hline \multirow{2}{*}{ PMMA } & DMF & $143-1505$ & 18 & {$[47]$} \\
\cline { 2 - 5 } & Ethyl acetate & $3840-3890$ & 2 & {$[70]$} \\
\hline PVDF & DMAA & 400 & 1 & {$[71]$} \\
\hline
\end{tabular}

Numerical data for fibre diameters were taken directly from the references. Where ranges or histograms were given mid points or distribution peaks were used to approximate averages. Some of the data included fibres with a variety of morphologies, including beads and ribbons; however the majority were reported to be smooth fibres. Due to the wide variety of measurement methods [72], and the relatively high standard deviations and skewed distributions typical in electrospun fibre mats [63], the error associated with the measured average diameter data is expected to be high.

Table 4. Data from literature used to train and test the ANN classification models.

\begin{tabular}{|c|l|c|l|}
\hline Polymer & \multicolumn{1}{|c|}{ Solvent } & Data points & Reference \\
\hline PS & DMF & 26 & {$[55]$} \\
\hline PVOH & Water & 34 & {$[33,66,73]$} \\
\hline \multirow{4}{*}{$P E O$} & Water & 22 & {$[46]$} \\
\cline { 2 - 4 } & DMF & 4 & {$[29]$} \\
\cline { 2 - 4 } & Ethanol & 4 & {$[29]$} \\
\cline { 2 - 4 } & Chloroform & 4 & {$[29]$} \\
\hline \multirow{3}{*}{$P$ LA } & DCM & 18 & {$[67]$} \\
\cline { 2 - 4 } & Acetone & 6 & {$[31]$} \\
\hline \multirow{3}{*}{$P V B$} & Ethanol & 6 & {$[68]$} \\
\cline { 2 - 4 } & Isopropanol & 6 & {$[68]$} \\
\cline { 2 - 4 } & n-Butanol & 6 & {$[68]$} \\
\hline$P A 6$ & Formic acid & 21 & {$[49]$} \\
\hline PA 66 & Formic acid & 43 & {$[69]$} \\
\hline PAN & DMF & 51 & {$[34,35]$} \\
\hline
\end{tabular}

Three classes (fibres, beaded fibres and no fibres) are used to classify the data in Table 4 . As it is impossible to define exact boundaries between what constitutes a beaded fibre versus a smooth fibre there is inherently some error to this data. This is due to the difficulties of examining a truly representative quantity of fibre with the limited field of vision available when using a microscope.

The data taken from these sources is also biased as researchers are more likely to present conditions leading to fibres rather than no fibres. To balance the bias extra data points will be generated for no fibre conditions. This can easily be done by combining non-compatible polymer/solvent combinations or by reducing the polymer concentration to zero.

The parameter data used to train the predictive models contains significant errors. A moderate source of error is polymer concentration. Most authors state the percentage weight to volume $\left(\%_{w / v}\right)$ which is calculated to one significant figure. Solutes are usually dissolved with continuous mixing, allowing significant solvent loss, particularly if the solvent is volatile. The authors own experience has shown concentration routinely varies up to $0.5 \%$ during this process. It is also common to start with a high concentration master batch and dilute to lower concentrations compounding the error. 
As concentration is expected to play a significant role in the determination of fibre diameter the effects of these errors will be particularly high. The dielectric constant data also contains significant error as it was not always possible to find values for polymers with the same molecular weight as those used in the experiments. In these cases interpolation was used.

\subsection{Data subsets}

Within the data outlined above, subsets were also created to compare how data containing solution measurements compared with the same data without the solution measurements. This will provide an idea of how much accuracy is lost by using only a priori information.

Data subsets will also be created to compare individual polymers with the generalised case.

In order to train, test and validate the predictive models each data subset was randomly partitioned into training, testing and validation sets with respective ratios of 70:20:10. The training data was used to train the ANNs while the testing data was used to select the highest performing networks. The validation data was used to assess how well the model generalises for new 'unseen' electrospinning conditions.

\section{Predictive models:}

Linear regression and ANNs were used to create the predictive models. The models were trained using Palisade's NeuralTools add in for Excel. Results from the highest performing models will be discussed.

\subsection{Probabilistic Neural Networks (PNNs) and Generalised Regression Neural Networks (GRNNs)}

PNNs are used for classification while GRNNs are often used for function approximation and prediction [74]. All PNNs and GRNNs consist of four layers as described below:

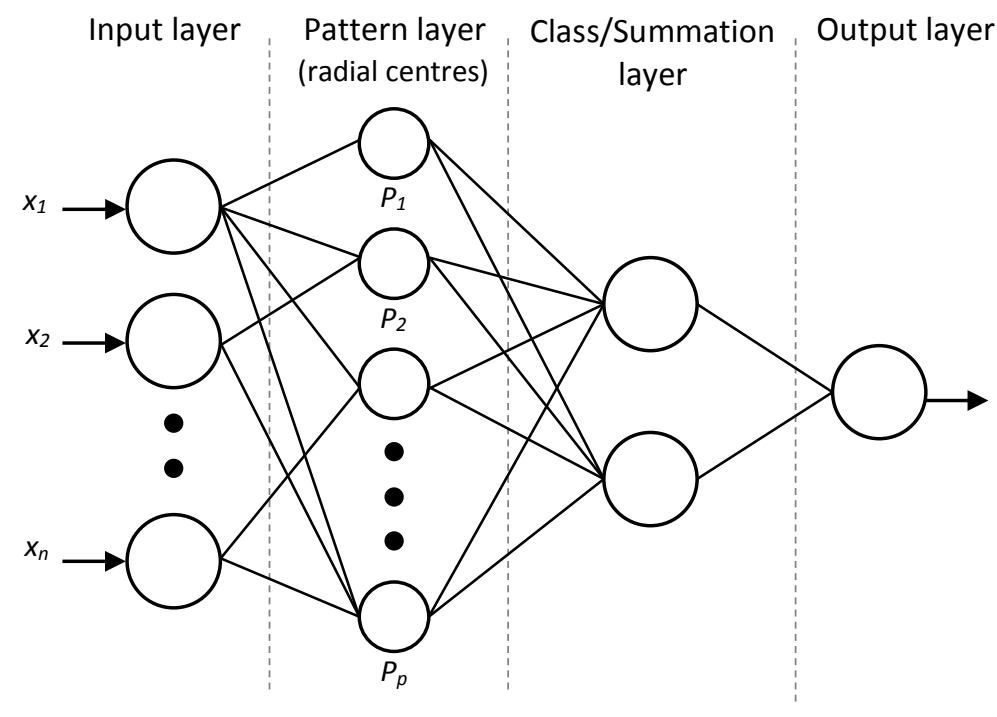

Figure 2. Architecture for a generalised regression neural network (GRNN).

- The input layer where the number of neurons equals the number of input parameters. 
- The pattern layer contains the radial centres for all the training points, it is also where the smoothing is applied to the radial basis function to minimise the predicted error.

- The third layer is different for PNNs and GRNNs. For PNNs it is a class layer containing one neuron for each class. For GRNNs it consists of two neurons, one neuron is the numerator the other is the denominator. The denominator adds up the weight values coming from each of the hidden neurons. The numerator adds up the weight values multiplied by the actual target value for each pattern neuron.

- $\quad$ For PNNs the output is the probability for each class. For GRNNs the output neuron divides the numerator by the denominator to produce the predicted value.

The PNN/GRNNs are trained by optimising the smoothing factor assigned to each training data point's radial basis function using the conjugate gradient descent method.

Unlike linear regression, ANNs can model highly nonlinear relationships. PNN/GRNNs train almost instantly and they do not require decisions about the system topology i.e. number of neurons or hidden layers. One disadvantage is that they do not extrapolate well outside the training data range. To mitigate this disadvantage a wide range of polymers and solvents have been included in the datasets.

\subsection{Multi-Layer Feed-forward Neural Network (MLFN)}

MLFN, also known as multilayer perceptrons, are also used for classification, function approximation and prediction [75]. The MLFNs used in this work contained one hidden layer, while the numbers of hidden neurons were varied to find an optimal topology (Figure 3):

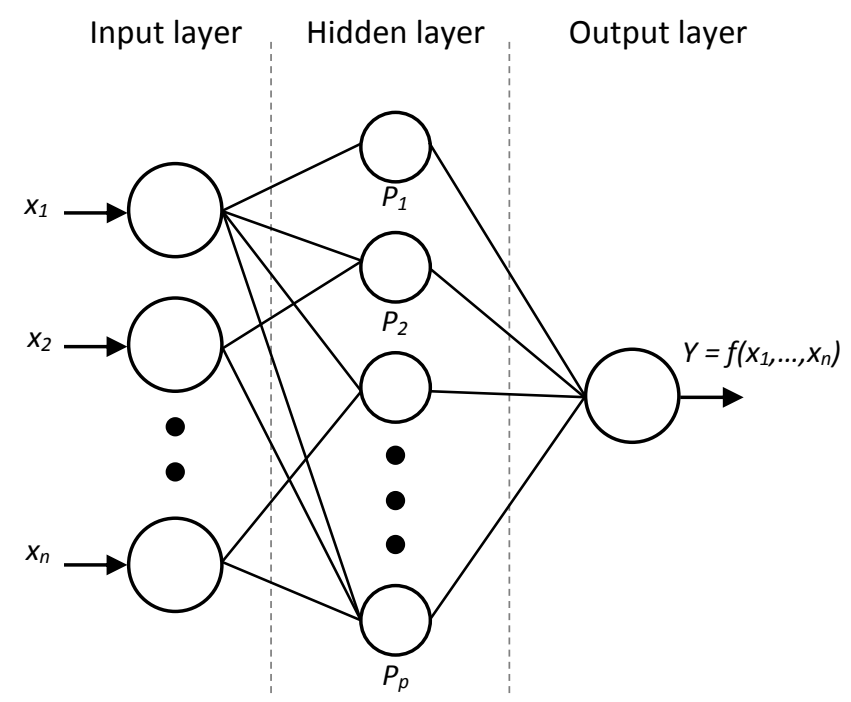

Figure 3. Architecture of a multilayer neural network with one hidden layer.

- The input layer contains a number of neurons equal to the number of input parameters. Weights are applied to the inputs as they are 'feed forward' to the hidden neurons.

- $\quad$ At the hidden layer the weighted values are fed into a transfer functions which are then distributed to the output layer. Again weights are applied to the values feed to the output layer. 
- Weighted values from the hidden layer arrive at the output neuron and are combined before entering another transfer function. The output of the transfer function is the output of the network.

The MLFNs were trained by optimising the weights using the conjugate gradient descent method[76]. Training was stopped when the reduction in error dropped below $1 \%$ in a 6 minute period.

\section{Results and discussion}

For each data subset statistical methods were used to find empirical predictive models. For the MFLNs optimal topologies were found by varying the number of neurons in the hidden layer between two and six. Trials using more than six neurons showed no improvement due to over fitting.

The results are split into two sections, spinning classification and fibre diameter prediction.

\subsection{Spinning classification}

The purpose of the classification model was to try and predict whether single needle electrospinning will occur given a polymer/solvent/concentration combination and what morphology the fibre will have. The classes are categorised as no fibres (electrospraying), beaded fibres and fibres. PNN's and MLFNs were trained using three sets of data; one larger set containing only easily known parameters and two smaller sets which were used to compare the performance of ANNs trained with and without solution measurements such as viscosity, surface tension and conductivity.

\subsubsection{Classification without solution measurements}

Initially all the parameters in table 2 were used to train 10 ANNs and a parameter impact analysis was carried out for each. Each ANN was trained with randomly selected testing data in order to mitigate data sensitivity on the impact analysis. The results were collated and parameters ranked in order of impact. The findings largely agreed with literature and are shown in Table 4.

Table 4. Impact of parameters on classification from impact analysis.

\begin{tabular}{|c|l|}
\hline $\begin{array}{c}\text { Impact on } \\
\text { classification }\end{array}$ & \multicolumn{1}{c|}{ Parameter } \\
\hline Strong & $\begin{array}{l}\text { Polymer concentration, Solvent density, Solvent viscosity, Polymer } \\
\text { molecular weight }\end{array}$ \\
\hline Moderate & $\begin{array}{l}\text { Polymer glass transition temperature, Electric field strength, Solvent } \\
\text { boiling point, Polymer dielectric constant }\end{array}$ \\
\hline Weak & $\begin{array}{l}\text { Solvent dielectric constant, Solvent vapour pressure, Solvent dipole } \\
\text { moment }\end{array}$ \\
\hline
\end{tabular}

To test the validity of the impact groupings shown above the ANNs were retrained without the 'weak' parameters. The performance of the ANNs trained with and without the weak parameters is shown below. The results are from the highest performing ANNs which were PNNs. The results (Table 5) show that removing the weak parameters slightly improved the performance of the PNN (although this is within the sensitivity range of the dataset). 
Table 5. Percentage of wrong predictions with and without weak parameters used in the data.

\begin{tabular}{|c|c|c|c|}
\hline \multirow{2}{*}{ Data inputs } & \multicolumn{3}{|c|}{ \% of wrong predictions } \\
\cline { 2 - 4 } & Training & Testing & Validation \\
\hline All parameters & $7.4 \%$ & $17.5 \%$ & $23.3 \%$ \\
\hline Weak parameters removed & $5.2 \%$ & $14.0 \%$ & $19.2 \%$ \\
\hline
\end{tabular}

A further breakdown of the classification results for the PNN without weak parameters is shown below. Most of the error comes from trying to predict beaded fibres. This is likely to be because, in the parameter space, beading occurs on a continuum between electrospraying (no fibres) and smooth fibres.

Table 6. Breakdown of classification predictions.

\begin{tabular}{|c|c|c|c|c|c|}
\hline & & \multicolumn{3}{|c|}{ Predicted classes } & \multirow{2}{*}{ Wrong predictions \% } \\
\hline & & Beaded & Fibres & None & \\
\hline & \multicolumn{5}{|c|}{ Training data } \\
\hline \multirow{11}{*}{$\begin{array}{c}\text { Observed } \\
\text { classes }\end{array}$} & Beaded & 35 & 3 & 2 & $12.5 \%$ \\
\hline & Fibres & 4 & 126 & 0 & $3.1 \%$ \\
\hline & None & 2 & 1 & 57 & $5.0 \%$ \\
\hline & \multicolumn{5}{|c|}{ Testing data } \\
\hline & Beaded & 5 & 3 & 0 & $37.5 \%$ \\
\hline & Fibres & 2 & 26 & 0 & $7.1 \%$ \\
\hline & None & 1 & 2 & 18 & $14.3 \%$ \\
\hline & \multicolumn{5}{|c|}{ Validation data } \\
\hline & Beaded & 2 & 1 & 0 & $33.0 \%$ \\
\hline & Fibres & 2 & 15 & 0 & $11.8 \%$ \\
\hline & None & 2 & 1 & 8 & $27.0 \%$ \\
\hline
\end{tabular}

The results show that it is possible to predict with over $80 \%$ accuracy whether smooth fibre electrospinning will occur, and if beading is present, using only readily available data known a priori. This result is significant as it could reduce the amount of time spent doing scoping exercises for new polymer/solvent systems.

In the next section a subset of data is used to test whether adding solution measurements such as viscosity improves the performance of the model.

\subsubsection{Classification with solution measurements}

The following table summarises how the performance of the networks changes if solution viscosity, conductivity and surface tension measurements were included.

Table 7. Percentage of wrong predictions with and without solution measurements.

\begin{tabular}{|c|c|c|c|}
\hline \multirow{2}{*}{ Data inputs } & \multicolumn{3}{|c|}{ \% of wrong predictions } \\
\cline { 2 - 4 } & Training & Testing & Validation \\
\hline $\begin{array}{c}\text { With solution } \\
\text { measurements }\end{array}$ & $0 \%$ & $8.3 \%$ & $7.7 \%$ \\
\hline
\end{tabular}




\begin{tabular}{|c|c|c|c|}
\hline $\begin{array}{c}\text { Without solution } \\
\text { measurements }\end{array}$ & $12.4 \%$ & $16.7 \%$ & $15.4 \%$ \\
\hline
\end{tabular}

It can be seen that in this case there is a reduction in wrong predictions if solution measurements are used, however this variation can easily be accounted for by the random selection of the testing data, as shown by the sensitivity analysis (Figure 4). The sensitivity of this dataset is high due to the low number of case points (134).

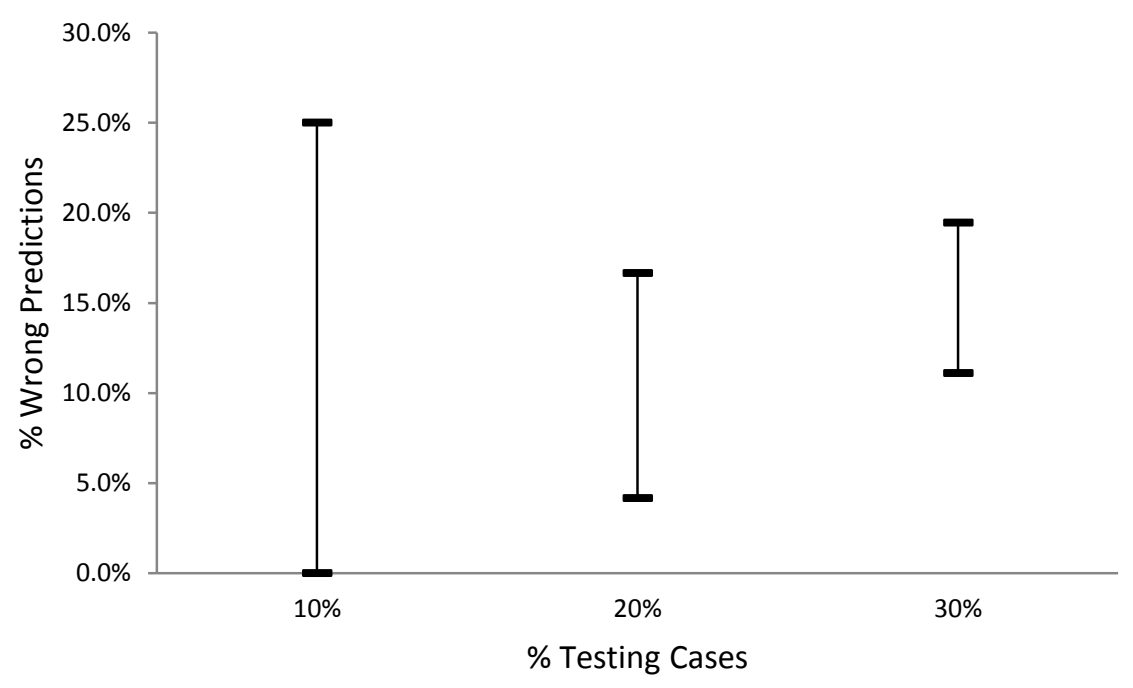

Figure 4. Sensitivity analysis showing the variation in prediction performance of ANN with solution measurements using randomly selected testing datasets and different dataset sizes.

Overall it is found that the performance gained by adding solution measurements is minimal and compromises the utility of a tool designed to reduce the time and cost of laboratory based electrospinning scoping exercises.

\subsection{Fibre diameter prediction}

The next question that might be asked after "Will it spin?" is "What will the fibre diameter be?" This section will present the results of ANNs used for fibre diameter prediction.

The performance measure used here will be the average absolute percentage error (average \% error) as it provides a more intuitive measure than the root-mean-square error (RMSE). Again the results will be based on three data sets; one containing all the fibre diameter cases and two smaller sets which were used to compare the performance of ANNs trained with and without solution measurements.

In all cases the ANNs significantly outperformed linear regression validating the claim that electrospinning phenomena is highly nonlinear relative to common input parameters.

\subsubsection{Diameter predictions without solution measurements}


The predictive model in this section was trained using polymer, solvent and experimental setup data with no knowledge of solution properties. Parameter impact analysis was used to determine the significance of the parameters in Table 2 . The results are summarised below.

Table 8. Impact of parameters on fibre diameter from impact analysis.

\begin{tabular}{|c|l|}
\hline $\begin{array}{c}\text { Impact on diameter } \\
\text { prediction }\end{array}$ & \multicolumn{1}{c|}{ Parameter } \\
\hline Strong & $\begin{array}{l}\text { Polymer concentration, Solvent viscosity, Polymer glass transition } \\
\text { temperature, Polymer dielectric constant, Polymer molecular weight }\end{array}$ \\
\hline Moderate & Electric field strength, Solvent density, Solvent boiling point \\
\hline Weak & $\begin{array}{l}\text { Solvent vapour pressure, Solvent surface tension, Solvent dielectric } \\
\text { constant, Solvent conductivity, Solvent dipole moment }\end{array}$ \\
\hline
\end{tabular}

Interesting to note is the strong impact the polymer glass transition temperature and dielectric constant have on diameter prediction. The physical relationship between the dielectric constant and fibre diameter is unknown, however it is unlikely to be related to solubility as the solvent dielectric constant has negligible impact.

A comparison was made for networks trained with and without the weak parameters confirming the network could perform equally well without them. Figure 5 below shows the predicted versus measured fibre diameters for the best performing ANN (a GRNN without the weak parameters). The training, testing and validation data all show a high level of correlation over a wide range of diameters (note the logarithmic scale). The average \% error for the training, testing and validation sets are $19.9 \%, 35.0 \%$ and $22.3 \%$ respectively which is comparable to the estimated errors of the input data. 


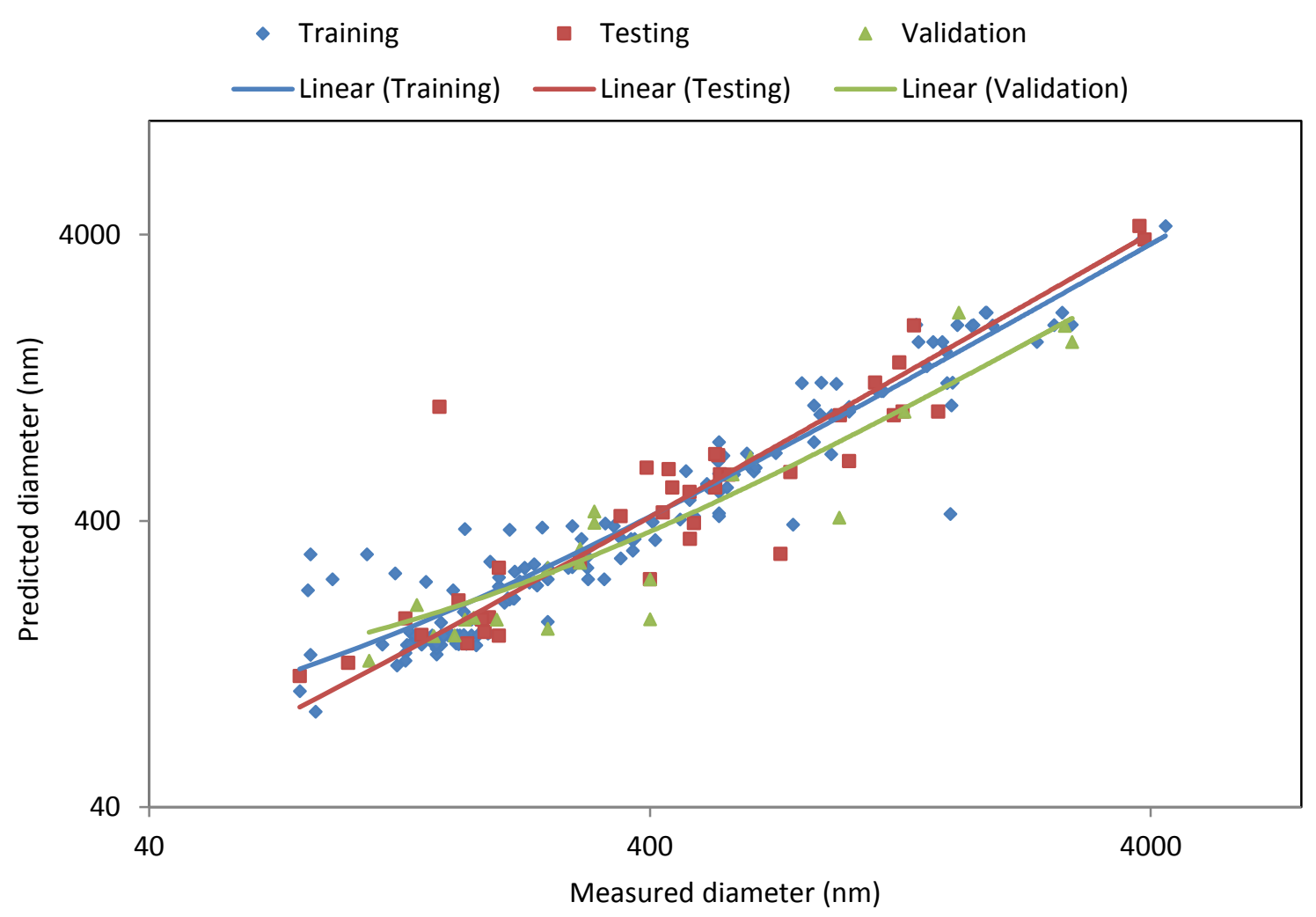

Figure 5. Predicted versus measured diameter for a GRNN trained using data for 13 different polymer/solvent combinations from over 20 independent studies.

Despite the exclusion of many parameters such as atmospheric conditions, needle diameter, flow rate, surface tension, vapour pressure, conductivity, collector geometry and more, it is striking to note that the average \% error for the validation data is only $22.3 \%$ or a RMSE of $186.8 \mathrm{~nm}$. This combined with the knowledge that the diameter measurements came from over twenty different studies all using different measurement methods shows that ANNs are a robust method for electrospinning predictions.

\subsubsection{Diameter predictions using solution measurement data}

The following figures show the predicted versus measured diameters for ANNs trained with a smaller dataset, with and without solution measurements such as viscosity, surface tension and conductivity.
- Training
- Testing
$\Delta$ Validation
Linear (Training)
Linear (Testing)
_Linear (Validation)

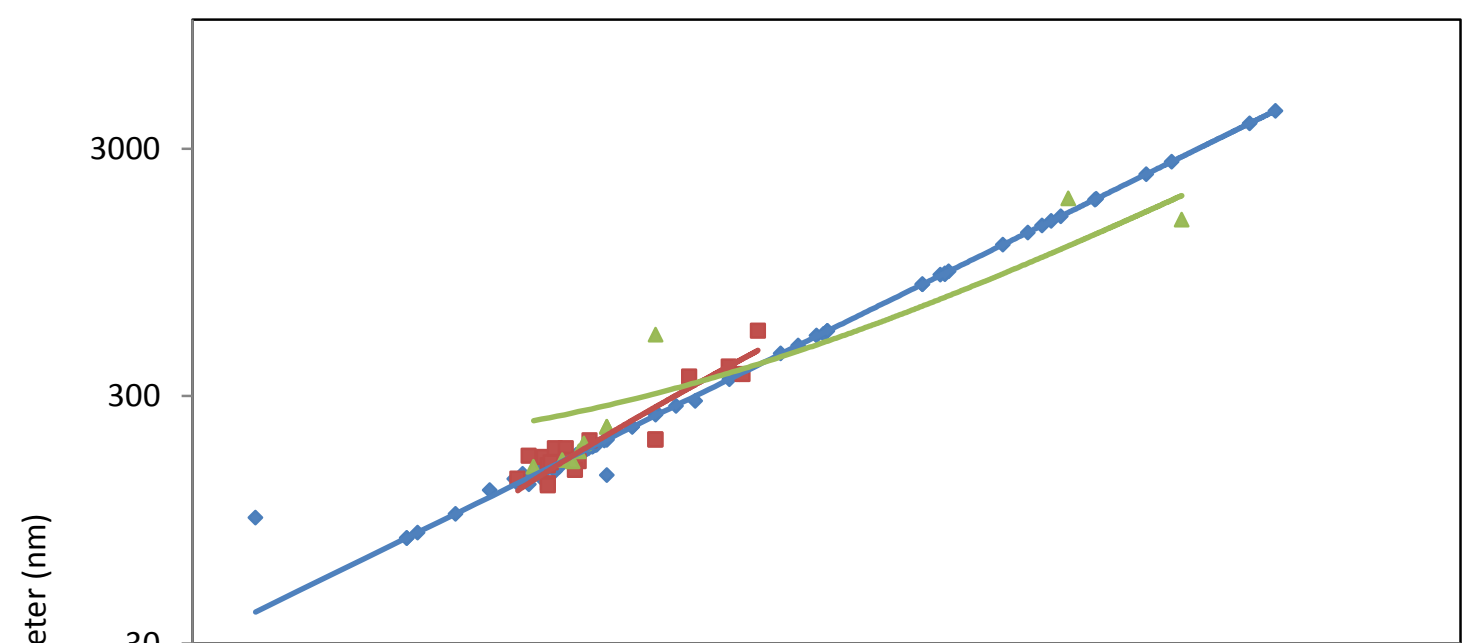




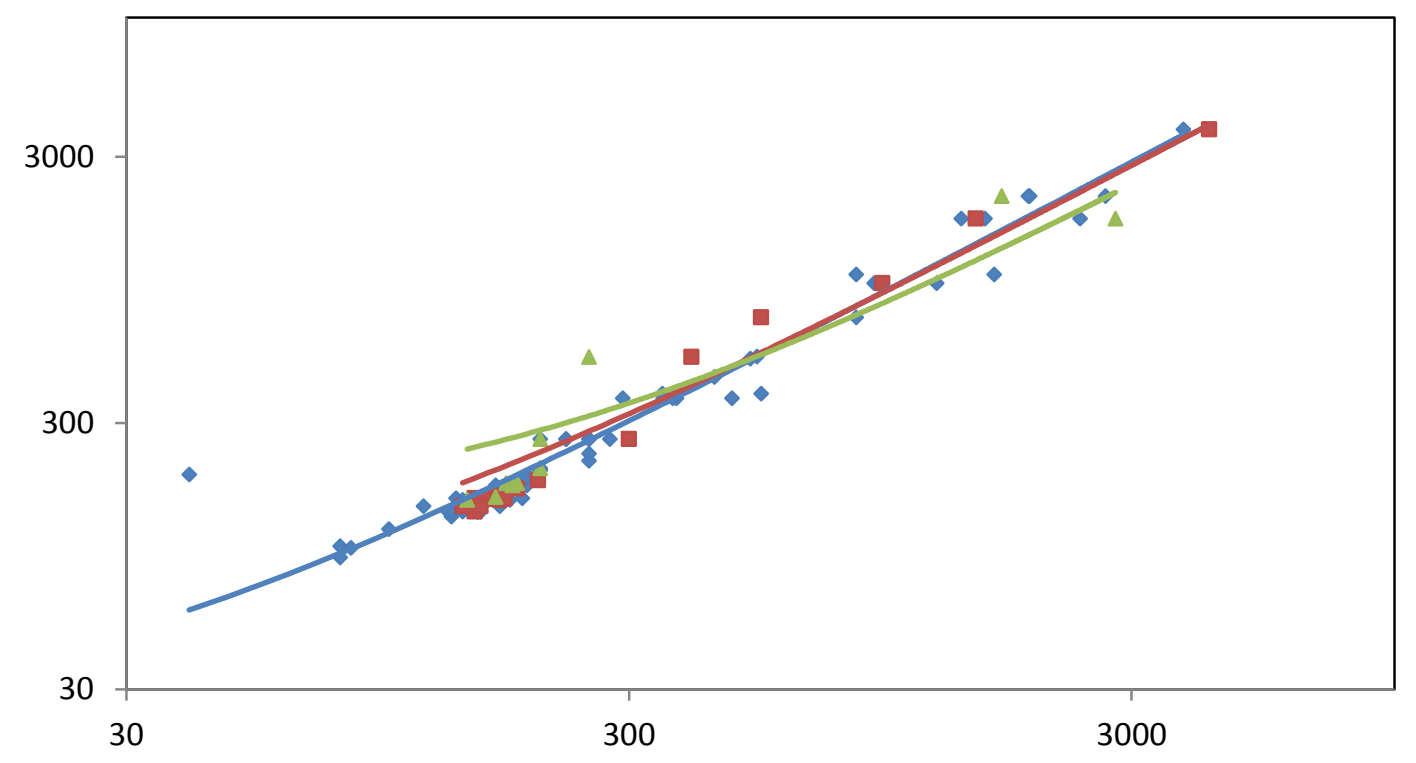

Figure 6. Predicted versus measured fibre diameters for ANNs trained with (top) and without (below) solution measurements.

The average \% error of the validation data with and without solution measurements is $22.0 \%$ and $22.7 \%$ respectively. These results validate the hypothesis that good predictions can be made without the need for solution measurements using only easily available prior knowledge.

\section{Conclusion}

The purpose of this work was to determine if ANNs could provide a generalised method of predicting electrospun fibre characteristics using only easily available, prior knowledge of the polymer, solvent, concentration and electric field strength. PNNs and GRNNs were found to outperform MLFNs and linear regression.

Trained PNNs were found to be effective at classifying fibre morphology and spin-ability with the validation data making over $80 \%$ correct predictions. Trained GRNNs were also effective at predicting fibre diameters with average $\%$ error at $22.3 \%$ for the validation data.

The inclusion of solution measurements was found to have a minimal impact on prediction performance. The parameter impact analyses for classification and diameter prediction is the largest quantitative study of this type known to the authors. The analysis helped to focus on which parameters influence important electrospinning characteristics, namely fibre morphology and 
diameter. In addition to this the success of the model goes someway to validate the exclusion of parameters such as feed rate and nozzle diameter.

The development of these tools will allow researchers to narrow down polymer, solvent and concentration combinations prior to carrying out time consuming scoping exercises. Further development of the tools by increasing the dataset size and reducing the errors associated with the input data may also allow for direct selection of optimised parameters for a given purpose. An interesting extension of this work would be the inclusion of fibre diameter standard deviation as a dependent value.

This paper used data from single needle setups with primary solvent systems and therefore cannot be used to predict needleless electrospinning or multiple solvent systems. Adapting the method for binary or ternary solvents will require further research.

\section{References}

1. Doshi J and Reneker DH. Journal of Electrostatics 1995;35(2-3):151-160.

2. Huang Z-M, Zhang YZ, Kotaki M, and Ramakrishna S. Composites Science and Technology 2003;63(15):2223-2253.

3. Yang F, Murugan R, Wang S, and Ramakrishna S. Biomaterials 2005;26(15):2603-2610.

4. Ayres C, Bowlin GL, Henderson SC, Taylor L, Shultz J, Alexander J, Telemeco TA, and Simpson DG. Biomaterials 2006;27(32):5524-5534.

5. Barnes CP, Sell SA, Boland ED, Simpson DG, and Bowlin GL. Advanced Drug Delivery Reviews 2007;59(14):1413-1433.

6. Sun T, Norton D, McKean RJ, Haycock JW, Ryan AJ, and MacNeil S. Biotechnology and Bioengineering 2007;97(5):1318-1328.

7. Ghasemi-Mobarakeh L, Prabhakaran MP, Morshed M, Nasr-Esfahani M-H, and Ramakrishna S. Biomaterials 2008;29(34):4532-4539.

8. Cantón I, McKean R, Charnley M, Blackwood KA, Fiorica C, Ryan AJ, and MacNeil S. Biotechnology and Bioengineering 2010;105(2):396-408.

9. Deshpande P, Ramachandran C, Sefat F, Mariappan I, Johnson C, McKean R, Hannah M, Sangwan VS, Claeyssens F, Ryan AJ, and MacNeil S. Biomaterials 2013;34(21):5088-5106.

10. Mota C, Puppi D, Gazzarri M, Bártolo P, and Chiellini F. Polymer International 2013;62(6):893-900.

11. Sefat F, McKean R, Deshpande P, Ramachandran C, Hill CJ, Sangwan VS, Ryan AJ, and MacNeil S. Procedia Engineering 2013;59(0):101-116.

12. Tamura T and Kawakami H. Nano Letters 2010;10(4):1324-1328.

13. Miao Y-E, Zhu G-N, Hou H, Xia Y-Y, and Liu T. Journal of Power Sources 2013;226(0):82-86.

14. Gopal R, Kaur S, Ma Z, Chan C, Ramakrishna S, and Matsuura T. Journal of Membrane Science 2006;281(1-2):581-586.

15. Barhate RS and Ramakrishna S. Journal of Membrane Science 2007;296(1-2):1-8.

16. Li D, Wang Y, and Xia Y. Advanced Materials 2004;16(4):361-366.

17. Chronakis IS. Journal of Materials Processing Technology 2005;167(2-3):283-293.

18. Teo WE and Ramakrishna S. Nanotechnology 2006;17(14):R89.

19. Cavaliere S, Subianto S, Savych I, Jones DJ, and Roziere J. Energy \& Environmental Science 2011;4(12):4761-4785.

20. Raghavan P, Lim D-H, Ahn J-H, Nah C, Sherrington DC, Ryu H-S, and Ahn H-J. Reactive and Functional Polymers 2012;72(12):915-930.

21. Agarwal S, Greiner A, and Wendorff JH. Progress in Polymer Science 2013;38(6):963-991. 
22. Mi Yeon S, Do Kyun K, Kyo Jin I, Seong Mu J, and Dong Young K. Nanotechnology 2004;15(12):1861.

23. Ondarçuhu T and Joachim C. EPL (Europhysics Letters) 1998;42(2):215.

24. Theron SA, Yarin AL, Zussman E, and Kroll E. Polymer 2005;46(9):2889-2899.

25. Zhou F-L, Gong R-H, and Porat I. Polymer International 2009;58(4):331-342.

26. Technology loN. Global Markets and Technologies for Nanofibers. 2013.

27. Yokoyama Y, Hattori S, Yoshikawa C, Yasuda Y, Koyama H, Takato T, and Kobayashi H. Materials Letters 2009;63(9-10):754-756.

28. Koski A, Yim K, and Shivkumar S. Materials Letters 2004;58(3-4):493-497.

29. Son WK, Youk JH, Lee TS, and Park WH. Polymer 2004;45(9):2959-2966.

30. Theron SA, Zussman E, and Yarin AL. Polymer 2004;45(6):2017-2030.

31. Cui W, Li X, Zhou S, and Weng J. Journal of Applied Polymer Science 2007;103(5):3105-3112.

32. Thompson CJ, Chase GG, Yarin AL, and Reneker DH. Polymer 2007;48(23):6913-6922.

33. Coles SR, Jacobs DK, Meredith JO, Barker G, Clark AJ, Kirwan K, Stanger J, and Tucker N. Journal of Applied Polymer Science 2010;117(4):2251-2257.

34. Yördem OS, Papila M, and Menceloğlu YZ. Materials \& Design 2008;29(1):34-44.

35. Gu SY, Ren J, and Vancso GJ. European Polymer Journal 2005;41(11):2559-2568.

36. Reneker DH, Yarin AL, Fong $\mathrm{H}$, and Koombhongse S. Journal of Applied Physics 2000;87(9):4531-4547.

37. Hohman MM, Shin M, Rutledge G, and Brenner MP. Physics of Fluids (1994-present) 2001;13(8):2201-2220.

38. Hohman MM, Shin M, Rutledge G, and Brenner MP. Physics of Fluids 2001;13(8):2221-2236.

39. Shin YM, Hohman MM, Brenner MP, and Rutledge GC. Polymer 2001;42(25):09955-09967.

40. Feng JJ. Journal of Non-Newtonian Fluid Mechanics 2003;116(1):55-70.

41. Han T, Yarin AL, and Reneker DH. Polymer 2008;49(6):1651-1658.

42. Rayleigh L. Proceedings of the Royal Society of London 1879;29:71-97.

43. Saville DA. Annual Review of Fluid Mechanics 1997;29(1):27-64.

44. Fong H, Chun I, and Reneker DH. Polymer 1999;40(16):4585-4592.

45. Helgeson ME and Wagner NJ. AIChE Journal 2007;53(1):51-55.

46. Sarkar K, Ghalia MB, Wu Z, and Bose SC. Journal of Materials Processing Technology 2009;209(7):3156-3165.

47. Khanlou H, Sadollah A, Ang B, Kim J, Talebian S, and Ghadimi A. Neural Computing and Applications 2014:1-11.

48. Nasouri K, Shoushtari A, and Khamforoush M. Fibers and Polymers 2013;14(11):1849-1856.

49. Ojha SS, Afshari M, Kotek R, and Gorga RE. Journal of Applied Polymer Science 2008;108(1):308-319.

50. Casper CL, Stephens JS, Tassi NG, Chase DB, and Rabolt JF. Macromolecules 2003;37(2):573578.

51. Gupta P, Elkins C, Long TE, and Wilkes GL. Polymer 2005;46(13):4799-4810.

52. Palangetic L, Reddy NK, Srinivasan S, Cohen RE, McKinley GH, and Clasen C. Polymer (0).

53. Luo CJ, Stride E, and Edirisinghe M. Macromolecules 2012;45(11):4669-4680.

54. Kim K, Kang M, Chin I-J, and Jin H-J. Macromolecular Research 2005;13(6):533-537.

55. Uyar T and Besenbacher F. Polymer 2008;49(24):5336-5343.

56. Biber E, Gündüz G, Mavis B, and Colak U. Applied Physics A 2010;99(2):477-487.

57. Macossay J, Marruffo A, Rincon R, Eubanks T, and Kuang A. Polymers for Advanced Technologies 2007;18(3):180-183.

58. Heikkilä $P$ and Harlin A. Express Polymer Letters 2009;3(7):437-445.

59. Stanger J, Tucker N, Wallace A, Larsen N, Staiger M, and Reeves R. Journal of Applied Polymer Science 2009;112(3):1729-1737.

60. Hardick O, Stevens B, and Bracewell D. Journal of Materials Science 2011;46(11):3890-3898. 
61. Luo X, Xie S, Liu J, Hu H, Jiang J, Huang W, Gao H, Zhou D, Lu Z, and Yan D. Polymer Chemistry 2014;5(4):1305-1312.

62. Deitzel J, Kleinmeyer J, Harris D, and Beck Tan N. Polymer 2001;42(1):261-272.

63. Yan X and Gevelber M. Journal of Electrostatics 2010;68(5):458-464.

64. Nurfaizey AH, Stanger J, Tucker N, Buunk N, Wallace A, and Staiger MP. Journal of Materials Science 2012;47(3):1156-1163.

65. Smallwood IM. Handbook of Organic Solvent Properties. In: Smallwood IM, editor. Oxford: Butterworth-Heinemann, 1996.

66. Tao J and Shivkumar S. Materials Letters 2007;61(11-12):2325-2328.

67. Zeng J, Haoqing H, Schaper A, Wendorff Joachim H, and Greiner A. Poly-L-lactide nanofibers by electrospinning - Influence of solution viscosity and electrical conductivity on fiber diameter and fiber morphology. e-Polymers, vol. 3, 2003. pp. 102.

68. Yener $\mathrm{F}$ and Jirsak O. Irainian Journal of Chemistry and Chemical Engineering (IJCCE) 2012;31(4):49-58.

69. Guerrini LM, Branciforti MC, Canova T, and Bretas RES. Materials Research 2009;12:181-190.

70. Li L, Li R, Li M, Rong Z, and Fang T. RSC Advances 2014;4(53):27914-27921.

71. Choi S-S, Lee YS, Joo CW, Lee SG, Park JK, and Han K-S. Electrochimica Acta 2004;50(23):339-343.

72. Stanger JJ, Tucker N, Buunk N, and Truong YB. Polymer Testing (0).

73. Keun Son W, Ho Youk J, Seung Lee T, and Park WH. Materials Letters 2005;59(12):15711575.

74. Specht DF. Neural Networks, IEEE Transactions on 1991;2(6):568-576.

75. Hornik K. Neural Networks 1991;4(2):251-257.

76. Hestenes MR and Stiefel E. Methods of conjugate gradients for solving linear systems: National Bureau of Standards Washington, DC, 1952. 